\title{
Continuous Blood Glucose Monitoring Outcomes in Veterans With Type 2 Diabetes
}

\author{
Sarah N. Langford, PharmD; Matthew Lane, PharmD; and Dennis Karounos, MD
}

Background: Nearly $25 \%$ of patients served in the US Department of Veterans Affairs have been diagnosed with type 2 diabetes mellitus (T2DM). Patients with DM typically monitor their blood glucose using intermittent fingerstick selftesting. Continuous glucose monitoring (CGM) might offer improved disease management.

Methods: We conducted a retrospective of VA patient records using a pre-post model. Average hemoglobin $A_{10}$ $\left(\mathrm{HbA}_{1 \mathrm{c}}\right)$ values were calculated for the year before and the year after CGM initiation. Our primary objective was to determine change in $\mathrm{HbA}_{1 \mathrm{c}}$ from the year before CGM initiation to the year after. Secondary objectives included changes in blood pressure, weight, and DM-related hospital and clinic visits during the same time frame.

Results: Both the total population and the adherent subgroup showed reduction in $\mathrm{HbA}_{1 \mathrm{c}}$. The complete population showed a $\mathrm{HbA}_{1 \mathrm{c}}$ change of -0.3 , and the adherent subgroup had a change of -1.3 . The total population had a mean change in weight of $-1.9 \mathrm{lb}(-0.9 \mathrm{~kg})$, and the adherent subgroup had an average change of $-8.0 \mathrm{lb}$. Average systolic blood pressure changes were $-0.1 \mathrm{~mm} \mathrm{Hg}$ in the total population and $+3.3 \mathrm{~mm} \mathrm{Hg}$ in the adherent subgroup. A decrease in total encounters for DM complications was observed in the total population ( -0.3 total encounters per patient) and the adherent subgroup ( -0.6 total encounters per patient).

Conclusions: CGM did not correspond with clinically significant reductions in $\mathrm{HbA}_{1 \mathrm{c}}$. However, veterans with increased health care engagement were likely to achieve clinically significant $\mathrm{HbA}_{1 \mathrm{c}}$ improvements. Adherent patients also had more reduction in weight and hospital or clinic visits with CGM compared with the total population.

\begin{abstract}
Sarah Langford is a PGY-1 Pharmacy Resident at St. Joseph Mercy Hospital in Ann Arbor, Michigan. Matthew Lane is Associate Professor and Pharmacy Residency Program Director in the College of Pharmacy, and Dennis Karounos is Associate Professor in the College of Medicine, all at University of Kentucky. Matthew Lane is Associate Chief of Pharmacy and Dennis Karounos is Director of Endocrinology Services, both at Lexington Veterans Affairs Health Care System in Kentucky.

Correspondence:

Sarah Langford

(langford.sarah@yahoo.com)
\end{abstract}

Fed Pract. 2021;38(suppl 4). Published online November 16. doi:10.12788/fp.0189
$\mathrm{N}$ early $25 \%$ of patients served in the US Department of Veterans Affairs (VA) have been diagnosed with type 2 diabetes mellitus (T2DM), although the prevalence among adults in the United States is $9 \% .{ }^{1} \mathrm{~Pa}$ tients with DM typically monitor their blood glucose using intermittent fingerstick selftesting. Continuous glucose monitoring (CGM) might offer a more comprehensive picture of glucose control to improve disease management. Within the VA, criteria for CGM use varies among facilities, but generally veterans prescribed at least 3 daily insulin injections and 4 daily blood glucose checks qualify.

CGM therapy has been extensively researched for type 1 DM (T1DM); however, outcomes of CGM use among older adults with T2DM have not been fully evaluated. In a 2018 review of randomized clinical trials evaluating CGM use, 17 trials examined only patients with T1DM (2009 participants), 4 included only patients with T2DM patients (547 patients), 3 evaluated patients with T1DM or T2DM (655 patients), and 3 included women with gestational diabetes (585 patients). ${ }^{3}$ Of 27 studies that included change in hemoglobin $\mathrm{A}_{1 \mathrm{c}}\left(\mathrm{HbA}_{1 \mathrm{c}}\right)$ as an endpoint, 15 found a statistically significant reduction in $\mathrm{HbA}_{1 \mathrm{c}}$ for the CGM group. Four trials evaluated CGM use in adults with T2DM and 3 found no difference in $\mathrm{HbA}_{1 \mathrm{c}}$ overall. However, 1 study found a difference in $\mathrm{HbA}_{1 \mathrm{c}}$ only in individuals aged $<65$ years, and another study found a greater improvement in the CGM group (approximately $0.5 \%$ ). 4,5 These mixed results indicate a need for further subgroup analysis in specific populations to determine the optimal use of CGM in adults with T2DM. Although this study was not designed to measure changes in hypoglycemic episodes or the relative efficacy of different CGM products, it establishes a baseline from which to conduct additional research.

Our primary objective was to determine change in $\mathrm{HbA}_{1 \mathrm{c}}$ in each patient from the year before CGM initiation to the year after. Secondary objectives included changes in blood pressure (BP), weight, and diabetes-related hospital and clinic visits during the same time frame. We also completed subanalysis comparing primary outcomes in engaged or adherent patients compared with the entire study group. This study was completed as a quality improvement project with approval from the Lexington Veterans Affairs Health Care System in Kentucky information security office and was exempted from institutional review board review.

\section{METHODS}

This project was a retrospective evaluation using the VA database of patient records. Rather than using a control group, our study used a prepost model to determine the impact of CGM for each patient. For the primary outcome, average $\mathrm{HbA}_{1 \mathrm{c}}$ values were calculated for the year be- 
fore and year after CGM initiation. Hemoglobin and hematocrit values were included if reported within 3 months of the $\mathrm{HbA}_{1 \mathrm{c}}$ values to ensure validity of $\mathrm{HbA}_{1 \mathrm{c}}$ results. Average $\mathrm{HbA}_{1 \mathrm{c}}$ was $13.37 \mathrm{~g} / \mathrm{dL}$ (range, 10.5-17.3), and average hematocrit was $43.3 \%$ (range, 36-52). Change in average $\mathrm{HbA}_{1 \mathrm{c}}$ was recorded for each patient. Based on research by Taylor and colleagues, a change in $\mathrm{HbA}_{1 \mathrm{c}}$ of $0.8 \%$ was considered clinically significant for this project. ${ }^{6}$

Mean BP and weight were calculated for the years before and after CGM initiation. Only values for routine clinic visits were included; values taken during an acute health incident, inpatient stay, infusion clinic appointments, or home readings were excluded. Changes were recorded for each patient. Patient encounter notes were used to determine the number of DMrelated hospital, emergency department, and clinic visits, such as nephrology, podiatry, vascular medicine, or infectious disease clinic or inpatient encounters during the study period. Routine endocrinology or primary care visits were not included, and patient care notes were consulted to ensure that the encounters were related to a $\mathrm{DM}$ complication. The change in number of visits was calculated for each patient.

Adherence was defined as patients receiving active medication management, documented treatment regimen adherence, and $>4$ annual endocrinology clinic visits. Active medication management was defined as having $>1$ dosage or medication change for oral or noninsulin antihyperglycemics, initiation, or adjustment of insulin dosages according to the patient records. Treatment adherence was determined based on medication reconciliation notes and refill request history. Only endocrinology clinic visits at VA outpatient clinics were included.

\section{Study Population}

A sample of 166 patients was needed to detect an $\mathrm{HbA}_{1 \mathrm{c}}$ change of 0.8 per power analysis. The normal approximation method using the $z$ statistic was used, with 2-tailed $\alpha=0.05, \beta=0.05, E=0.8$, and $S=1.2$. We randomly selected 175 patients among all individuals with an active prescription for CGM in 2018 and 2019, who had a diagnosis of T2DM, and were managed by VA endocrinology clinics (including endocrine clinics, diabetes clinics, and patient aligned care team clinics) at 87 VA medical centers. Patients with types of DM other than T2DM were excluded, as well as those with a diagnosed hemoglobinopathy or
TABLE 1 Baseline Demographics

\begin{tabular}{|c|c|c|}
\hline Characteristics & Total $(\mathrm{N}=175)$ & Adherent Group $(n=40)$ \\
\hline \multicolumn{3}{|l|}{ Race/ethnicity, No. (\%) } \\
\hline White & $129(73.7)$ & $36(90.0)$ \\
\hline Black/African American & 29 (16.6) & $3(7.5)$ \\
\hline Hispanic/Latino & $4(2.3)$ & $0(0)$ \\
\hline Asian & $6(3.4)$ & $1(2.5)$ \\
\hline Native/Pacific Islander & $4(2.3)$ & 0 \\
\hline Age, mean (SD), y & $69.0(9.5)$ & $65.5(5.3)$ \\
\hline Sex, male, No. (\%) & $167(95.4)$ & $39(97.5)$ \\
\hline \multicolumn{3}{|l|}{ Baseline medication, No. (\%) } \\
\hline Oral or noninsulin only & $107(61.1)$ & $4(10.0)$ \\
\hline Insulin only & $4(2.3)$ & $1(2.5)$ \\
\hline Insulin + oral or noninsulin & 64 (36.6) & 35 (87.5) \\
\hline
\end{tabular}

$\mathrm{HbA}_{1 \mathrm{c}}<10 \mathrm{~g} / \mathrm{dL}$. The adherent subgroup included 40 patients of the 175 sample population (Table 1).

\section{RESULTS}

Both the total population and the adherent subgroup showed reduction in $\mathrm{HbA}_{1 c}$, the primary endpoint. The complete population showed a $\mathrm{HbA}_{1 \mathrm{c}}$ change of $-0.3(95 \% \mathrm{Cl},-0.4$ to -0.2$)$, and the adherent subgroup had a change of $-1.3(95 \% \mathrm{Cl},-1.5$ to -1.2$)$. The total survey population had a mean change in weight of $-1.9 \mathrm{lb}(-0.9 \mathrm{~kg})(95 \% \mathrm{Cl},-3.7$ to -0.1$)$ and the adherent subgroup had an average change of $-8.0 \mathrm{lb}(-3.6 \mathrm{~kg})(95 \% \mathrm{Cl},-12.3$ to -3.8$)$. Average systolic BP changes were $-0.1 \mathrm{~mm} \mathrm{Hg}$ $(95 \% \mathrm{Cl},-1.6$ to 1.5$)$ in the total population and $+3.3 \mathrm{~mm} \mathrm{Hg}(95 \% \mathrm{Cl},-0.01$ to 6.22$)$ in the adherent subgroup. A decrease in total encounters for DM complications was observed in the population ( -0.3 total encounters per patient, $95 \% \mathrm{Cl},-0.5$ to -0.2$)$ and the adherent subgroup ( -0.6 total encounters per patient, 95\% $\mathrm{Cl},-1.0$ to -0.1 ) (Table 2).

Before the study, 107 (61.1\%) patients were taking oral or noninsulin DM medication only, $4(2.3 \%)$ were on insulin only, and 64 (36.6\%) were prescribed both insulin and oral/noninsulin antihyperglycemics. Noninsulin and oral antihyperglycemic regimens included combinations of biguanide, dipeptidyl peptidase4 inhibitor, sodium-glucose cotransporter-2 inhibitor, sulfonylurea, meglitinide, $\beta$-glucosidase inhibitor, glucagon-like peptide-1 (GLP-1) analog, and thiazolidinedione drug classes. Nearly $70 \%$ (122) had no reported changes in DM treatment beyond dosage titrations. Among these patients, 18 (10.3\%) were on an insulin pump for the 
TABLE 2 Patients Using CGM

\begin{tabular}{|c|c|c|c|}
\hline Criteria & $\begin{array}{c}\text { Before CGM, } \\
\text { mean (SD) }\end{array}$ & $\begin{array}{l}\text { After CGM, } \\
\text { mean (SD) }\end{array}$ & $\begin{array}{c}\text { Change, } \\
\text { mean }(95 \% \mathrm{Cl})\end{array}$ \\
\hline \multicolumn{4}{|l|}{ Hemoglobin $\mathrm{A}_{1}, \%$} \\
\hline Total population & $8.1(1.21)$ & $7.8(1.2)$ & $-0.3(-0.4,-0.2)$ \\
\hline Adherent subgroup & $8.9(1.03)$ & $7.6(1.0)$ & $-1.3(-1.5,-1.2)$ \\
\hline \multicolumn{4}{|l|}{ Weight, kg } \\
\hline Total population & $98.0(54.0)$ & $97.1(51.7)$ & -0.9 \\
\hline Adherent subgroup & $99.4(48.5)$ & $95.8(44.8)$ & -3.6 \\
\hline \multicolumn{4}{|c|}{ Systolic blood pressure, $\mathrm{mm} \mathrm{Hg}$} \\
\hline Total survey population & $132.5(11.5)$ & $132.5(12.1)$ & $-0.1(-1.6,+1.5)$ \\
\hline Adherent subgroup & $130.3(12.0)$ & $133.4(11.4)$ & $+3.1(-0.01,+6.22)$ \\
\hline \multicolumn{4}{|l|}{ Encounters per patient } \\
\hline Total population & $1.4(1.1)$ & $1.0(1.0)$ & $-0.3(-0.53,-0.2)$ \\
\hline Inpatient admissions & $0.1(0.3)$ & $0.03(0.2)$ & $-0.1(-0.4,+0.2)$ \\
\hline ED visits & $0.5(0.7)$ & $0.3(0.5)$ & $-0.2(-1.0,+0.7)$ \\
\hline Adherent subgroup & $1.6(1.4)$ & $1.0(1.1)$ & $-0.6(-1.0,-0.1)$ \\
\hline Inpatient admissions & $0.04(0.1)$ & $0.02(0.1)$ & $-0.01(-0.04,+0.02)$ \\
\hline ED visits & $0.2(0.2)$ & $0.1(0.2)$ & $-0.1(-0.10,-0.04)$ \\
\hline Total, No. & 236 & 176 & -61 \\
\hline Inpatient admissions & 16 & 6 & -10 \\
\hline ED visits & 79 & 53 & -26 \\
\hline Adherent subgroup, No. & 64 & 42 & -22 \\
\hline Inpatient admissions & 2 & 1 & -1 \\
\hline ED visits & 9 & 4 & -5 \\
\hline
\end{tabular}

Abbreviations: CGM, continuous glucose monitoring; ED, emergency department.

duration of the study. Among the 53 (30.3\%) patients who had changes in treatment, $31(17.7 \%)$ transitioned from insulin injections to an insulin pump, $13(7.4 \%)$ changed from 1 insulin injection to another (ie, addition of long-acting insulin, transition to u500 insulin, changing from 1 insulin category or brand to another), 8 (4.6\%) began an oral/noninsulin antihyperglycemic, 4 (2.3\%) began insulin injections, $13(7.4 \%)$ discontinued noninsulin or oral antihyperglycemics, and $2(1.1 \%)$ discontinued insulin during the study period.

Data showed that $113(64.5 \%)$ patients had no changes in antihypertensives. The remaining 62 (35.4\%) had the following adjustments: $14(8 \%)$ increased dose of current medication(s), 9 (5.1\%) decreased dose of current medication(s), 8 (4.6\%) discontinued all antihypertensive medications, 10 (5.7\%) switched to a different antihypertensive class, and $16(9.1 \%)$ added additional antihypertensive medication(s) to their existing regimen during the study period.

Patients in the study group used 7 different types of CGM sensors. Chart review revealed that $84(47.7 \%)$ patients used Medtronic devices, with 26 (14.8\%) using first-generation Guardian sensors, $50(28.4 \%)$ using Enlite sensors, and 8 (4.5) using Guardian 3 sensors. We found that $81(46.0 \%)$ veterans were prescribed Dexcom devices, with $5(2.8 \%)$ using SEVEN PLUS sensors, 68 (38.6\%) using G4-5 sensors, and $8(4.5 \%)$ using G6 sensors. The remaining 10 (5.7\%) patients were using Freestyle Libre sensors during the study period.

\section{DISCUSSION}

CGM did not correspond with clinically significant reductions in $\mathrm{HbA}_{1 c}$. However, veterans with increased health care engagement were likely to achieve clinically significant $\mathrm{HbA}_{1 c}$ improvements. The veterans in the adherent subgroup had a higher baseline $\mathrm{HbA}_{1 \mathrm{c}}$, which could be because of a variety of factors mentioned in patient care notes, including insulin resistance, poor dietary habits, and exercise regimen nonadherence. These patients might have had more room to improve their glycemic control without concern of hypoglycemia, and their higher baseline $\mathrm{HbA}_{1 \mathrm{c}}$ could have provided increased motivation for improving their health during the study period.

Adherent patients also had a greater reduction in weight and hospital or clinic visits with CGM compared with the total population. These veterans' increased involvement in their health care might have led to better dietary and exercise adherence, which would have decreased insulin dosing and contributed to weight loss. Only 1 patient in the adherent subgroup initiated a GLP-1 agonist during the study period, making it unlikely that medication changes had a significant impact on weight loss in the subgroup analysis. This improvement in overall health status might have contributed to the reduction in hospital or clinic visits observed in this population.

Average systolic BP data decreased minimally in the total survey population and increased in the adherent subgroup over the course of the study. These results were determined to be statistically significant. Changes in systolic BP readings were minimal, indicating that it is unlikely that these changes contributed meaningfully to the patients' overall health status.

Although not related to the study objectives, the adherent population required less antihypertensive adjustments with similar BP control. This could be explained by 
improved overall health or better adherence and engagement in therapy. The results of this project show that despite limited medication changes, T2DM management improved among adherent patients using CGM. The general study population, which was more likely to have documented nonadherence with treatment or clinic appointments, had minimal benefit. CGM technology in the T2DM veteran population is more likely to have significant clinical benefit in patients who are adherent with their medication regimens and follow-up appointments compared with the larger study population.

The results of this study are in line with previous studies on CGM use in the T2DM patient population. We agree with the previously published research that CGM alone does not have a meaningful impact on $\mathrm{HbA}_{1 \mathrm{c}}$ reduction. Our study population also was older than those in previous studies, adding to the Haak and colleagues conclusion that patients aged $<65$ years might have better outcomes with CGM. $^{4}$

Strengths of this study include specificity to the veteran population using VA resources, as well as including nondiabetes outcomes. This allows for specific application to the veteran population and could provide broader evidence for CGM use. Demonstrated decreases in $\mathrm{HbA}_{1 \mathrm{c}}$, weight, and clinic visits in the adherent population suggest that providing veterans with CGM therapy with frequent endocrinology follow-up improves health outcomes and could decrease overall health spending.

\section{Limitations}

Limitations of this study include retrospective design, a small sample size, and solely focusing on T2DM. As a retrospective study, we cannot rule out the influence of outside factors, such as participation in a non-VA weight loss program. This study lacked the power to assess the impact of the different CGM brands. The study did not include data on severe hypoglycemic or hyperglycemic episodes as veterans might have needed emergent care at non-VA facilities. Future research will evaluate the impact of CGM on symptomatic and severe hypoglycemic episodes and use of insulin vs oral or noninsulin antihyperglycemics and the comparative efficacy of different CGM brands among veterans.

\section{CONCLUSIONS}

CGM did not correspond with clinically significant reductions in $\mathrm{HbA}_{1 \mathrm{c}}$. However, veterans with increased health care engagement were likely to achieve clinically significant $\mathrm{HbA}_{1 \mathrm{c}}$ improvements. Adherent patients also had more reduction in weight and hospital or clinic visits with CGM compared with the total population. These veterans' increased involvement in their health care might have led to better dietary and exercise adherence, which would have decreased insulin dosing and contributed to weight loss.

\section{Author disclosures}

The authors report no actual or potential conflicts of interest with regard to this article.

\section{Disclaimer}

The opinions expressed herein are those of the authors and do not necessarily reflect those of Federal Practitioner, Frontline Medical Communications Inc., the US Government, or any of its agencies. This article may discuss unlabeled or investigational use of certain drugs. Please review the complete prescribing information for specific drugs or drug combinations-including indications, contraindications, warnings, and adverse effectsbefore administering pharmacologic therapy to patients.

\section{References}

1. Liu $Y$, Sayam S, Shao $X$, et al. Prevalence of and trends in diabetes among veterans, United States, 2005-2014. Prev Chronic Dis. 2017;14:E135. Published 2017 Dec 14. doi:10.5888/pcd14.170230

2. Hackett M. VA pharmacies now carry the Dexcom G6 CGM at no cost for qualifying patients. September 23, 2020. Accessed September 28, 2021. https://www .mobihealthnews.com/news/va-pharmacies-now-carry -dexcom-g6-cgm-no-cost-qualifying-patients

3. Peters AL. The evidence base for continuous glucose monitoring. In: Role of Continuous Glucose Monitoring in Diabetes Treatment. Arlington (VA): American Diabetes Association; August 2018.3-7. doi:10.2337/db20181-3

4. Haak T, Hanaire H, Ajjan R, Hermanns N, Riveline JP, Rayman G. Flash glucose-sensing technology as a replacement for blood glucose monitoring for the management of insulin-treated type 2 diabetes: a multicenter, open-label randomized controlled trial. Diabetes Ther. 2017;8(1):55-73. doi:10.1007/s13300-016-0223-6

5. Yoo HJ, An HG, Park SY, et al. Use of a real time continuous glucose monitoring system as a motivational device for poorly controlled type 2 diabetes. Diabetes Res Clin Pract. 2008;82(1):73-79. doi:10.1016/j.diabres.2008.06.015

6. Taylor PJ, Thompson CH, Brinkworth GD. Effectiveness and acceptability of continuous glucose monitoring for type 2 diabetes management: A narrative review. $J$ Diabetes Investig. 2018;9(4):713-725. doi:10.1111/jdi.12807 